\title{
An Enhanced Secure Remote User Authentication Scheme without Verification Table
}

\author{
Sumitra Binu \\ Research scholar \\ Christ University \\ Bangalore, India
}

\author{
Pethuru Raj, Ph.D \\ Cloud Solution Architect \\ IBM Global Cloud Center of \\ Excellence, \\ IBM India, Bangalore
}

\author{
M. Misbahuddin, Ph.D \\ Senior Technical Officer \\ C-DAC, Electronic City \\ Bangalore, India
}

\begin{abstract}
With the significant advances in communication networks over the last few decades, smart cards have been widely used in many e-commerce applications and network security protocols due to their low cost, portability, efficiency and cryptographic properties. In this paper, we analyze Sood et al.'s smart card based authentication scheme and demonstrate that the scheme is vulnerable to masquerade user attack, offline password guessing attack, time concurrency weaknesses and fails to achieve mutual authentication. A secure dynamic identity based remote user authentication scheme without verification tables, is proposed in this paper and the scheme resolves the aforementioned problems of Sood et al.'s scheme. The computation cost of the proposed scheme is comparable to Sood et al.'s scheme and it is highly secure taking into consideration the complexity of calculating discrete logarithms and the resistance to various attacks.
\end{abstract}

\section{General Terms}

Security

\section{Keywords}

Remote User Authentication, Smart Cards, No Verification Table, Cyclic groups, Discrete Logarithm

\section{INTRODUCTION}

Password based authentication protocols is the most commonly used mechanism carrying out remote user authentication. Many research works depicting the application of password-based authentication schemes with smart cards to verify the legitimacy of remote user login requests have already been published [1, 2, 3, 4, 5, 6, 7]. In 1981, Lamport [8] proposed a password based authentication scheme though it failed to preserve user anonymity. In 2004, Das et al., [9] proposed a user anonymity preserving, dynamic identity based remote user authentication scheme. Later the scheme was proved to be susceptible to insider attack, masquerade attack and server spoofing attack [10,11,12]. Chien and Chen [13] proposed an improvement of Das et al.'s scheme, but is highly computation intensive. In 2005, Liao et al., [14] proposed a scheme that enhances the security of Das et al.'s scheme and supports mutual authentication but Yoon and Yoo [15] demonstrated a reflection attack on their scheme. In 2006, Liou et al., [16] suggested a new dynamic identity based remote user mutual authentication scheme and Shih [17] demonstrated that their scheme fails to achieve mutual authentication. Hsiang and Shih in 2009[18] proposed a remote user authentication scheme. This scheme was found to be susceptible to server spoofing attack, replay attack and password guessing attackby Sood et al. [19]. Again in 2010, Sood et al. [19] proved that Liou et al.'s scheme is susceptible to $\mathrm{Ku}$ et al.'s impersonation attack [20] and proposed a modified scheme. Kwang Cheul Shin and Jung Gil Cho[21] pointed out that Sood et al.'s scheme is vulnerable to steal information from database attack, insider attack etc. In this paper, it is demonstrated that Sood et al.'s scheme is also susceptible to masquerade user attack, database attack etc. and propose a modified version which resolves the weaknesses of Sood et al.'s scheme. The discussed scheme preserves user anonymity and does not require the server to maintain a verification table which in turn resists the stolen verifier attack

The rest of the paper is organized as follows. In section 2, a brief overview of Sood et al.'s scheme [19] is given. Section 3 describes the weaknesses of Sood et al.'s scheme. Section 4, discusses the proposed scheme and security analysis of the scheme is presented in Section 5. Cost and functionality comparison is shown in section 6 and section 7 concludes the work done.

\section{REVIEW OF SOOD ET AL.'S SCHEME}

This section includes a brief overview of Sood et al.'s scheme. The scheme involves two participants' viz., the user U and the Server S. There are four phases in this scheme which includes the Registration, login, authentication and password change phase. During the registration phase a user submits his identity, ID and password, PW to S. S computes the security parameter's using his secret key $\mathrm{x}$ and random value $\mathrm{y}$, stores the same in a smart card (SC) and sends it to the user U via a secure channel. During the login phase, U inserts the SC and types in his ID and PW. The SC verifies the authenticity of the user by checking the password and then creates the login request. The request is sent by $U$ to $S$. During the authentication phase, $U$ and $S$ mutually authenticate each other and the password change phase allows the user to change the password without the intervention of S. To shorten the length of the paper, we omit the review. Please refer to [19] for Sood et al.'s protocol.

\section{WEAKNESSES OF SOOD ET AL.'S SCHEME}

Kwang Cheul Shin and Jung Gil Cho[21] pointed out that Sood et al.'s scheme is vulnerable to stolen verifier attack, insider attack etc. This section demonstrates that the protocol is also susceptible to impersonation attack, time concurrency weaknesses and fails to preserve user anonymity.

\subsection{Offline Secret Key Guessing Attack}

A valid but malicious user can extract the security parameters $B_{i}, C_{i}$ and $D_{i}$ stored in the memory of his smart card and can extract $h(x)$ from $D_{i}$ using his own $\operatorname{ID}_{i}$ and $P_{i}$. Then the adversary can try to guess different values of $x$ and check its correctness by verifying it with the value of $\mathrm{h}(\mathrm{x})$. 


\subsection{Time Concurrency Weakness}

Sood et al.'s scheme uses time concurrency mechanism to resist replay attacks possible during an authentication session. Studies shows that the usage of time stamps are susceptible to certain drawbacks such as different time zones of Client and Server, delivery latency etc. [7]. The lack of synchronization of clocks of the participants can lead to the failure of the authentication scheme.

\subsection{Denial of Service Attack}

An adversary $\mathrm{U}_{\mathrm{k}}$, who gets hold of the smart card(SC) of a registered user $U_{i}$ can extract the parameters $B_{i}, C_{i}$ and $D_{i}$ using various side-channel attacks. Then $\mathrm{U}_{\mathrm{k}}$ modifies $\mathrm{B}_{\mathrm{i}}$ to $\mathrm{B}_{\mathrm{i}}{ }^{*}$. When $\mathrm{U}_{\mathrm{i}}$ tries to login later by using his $\mathrm{SC}$ and entering $\mathrm{ID}_{\mathrm{i}}$ and $\mathrm{P}_{\mathrm{i}}$, the $\mathrm{SC}$ computes $\mathrm{h}\left(\mathrm{x} \| \mathrm{y}_{\mathrm{i}}\right)^{*}=\mathrm{B}_{\mathrm{i}}{ }^{*} \oplus \mathrm{h}\left(\mathrm{ID}_{\mathrm{i}} \| \mathrm{P}_{\mathrm{i}}\right) \oplus$ $\mathrm{P}_{\mathrm{i}}$. Then SC calculates $\mathrm{C}_{\mathrm{i}}{ }^{*}=\mathrm{h}\left(\mathrm{x} \| \mathrm{y}_{\mathrm{i}}\right)^{*} \oplus \mathrm{h}\left(\mathrm{P}_{\mathrm{i}}\right)$ and compares $\mathrm{C}_{\mathrm{i}}{ }^{*}$ with $\mathrm{C}_{\mathrm{i}}$. There will be a mismatch since $\mathrm{B}_{\mathrm{i}}$ was modified and hence $U_{i}$ will be denied service. DOS to a valid user also happens when an administrator updates any secret information specific to a valid user, which is stored in the database of the server. In Sood et al.'s scheme, the server maintains a data base that stores the secret information of valid users. If a malicious administrator modifies this information, then a registered user will not be able to login to the server with his valid credentials.

\subsection{Masquerade Server Attack}

If a malicious user $U_{i}$ attempts to impersonate the server $S$, she must be able to forge a valid challenge $\left(V_{i}, T\right.$ "). $U_{i}$ can get $\mathrm{h}(\mathrm{x})$ from his own smart card. Then if he intercepts a legitimate user $\mathrm{U}_{\mathrm{k}}$ 's login request, he can obtain $\left(\mathrm{CID}_{\mathrm{k}}, \mathrm{M}_{\mathrm{k}}\right.$, $\mathrm{T})$. He calculates, $\mathrm{h}\left(\mathrm{x} \| \mathrm{y}_{\mathrm{k}}\right)=\mathrm{CID}_{\mathrm{k}} \oplus \mathrm{h}(\mathrm{h}(\mathrm{x}) \| \mathrm{T})$. Again, if $\mathrm{U}_{\mathrm{i}}$ intercepts the server's response $\left(\mathrm{V}_{\mathrm{i}}, \mathrm{T}^{\prime \prime}\right)$, he can generate his own version of the response as $\left(\mathrm{V}_{\mathrm{im}}, \mathrm{T}^{\prime \prime}\right)$ where $\mathrm{V}_{\mathrm{im}}=(\mathrm{h}(\mathrm{x} \|$ $\left.\left.\mathrm{y}_{\mathrm{k}}\right)\|\mathrm{h}(\mathrm{x})\| \mathrm{T} \| \mathrm{T}^{\prime \prime}\right)$ and send $\left(\mathrm{V}_{\mathrm{im}}, \mathrm{T}^{\prime \prime}\right)$ to the user $\mathrm{U}_{\mathrm{k}}$.

\subsection{Impersonation Attack}

In this attack a malicious user $U_{\mathrm{i}}$ tries to forge a valid login request message $\left(\mathrm{CID}_{\mathrm{V}}, \mathrm{M}_{\mathrm{V}}, \mathrm{T}\right)$ sent by the valid user where $\mathrm{CID}_{\mathrm{V}}, \mathrm{M}_{\mathrm{V}}$ are the CID and $\mathrm{M}$ values, of a valid registered user. Let us assume the following attack scenario.

The malicious valid User $U_{i}$ has his own smart card from which he extracts $B_{i}, C_{i}$ and $D_{i}$. Computes $h(x)=D_{i} \oplus h\left(I_{i} \|\right.$ $\mathrm{P}_{\mathrm{i}}$ ) using his own $\mathrm{ID}_{\mathrm{i}}$ and $\mathrm{P}_{\mathrm{i}} \cdot \mathrm{h}(\mathrm{x})$ is common for all valid users. At the login phase, the valid user $\mathrm{U}_{\mathrm{v}}$ sends $\left(\mathrm{CID}_{\mathrm{V}}, \mathrm{M}_{\mathrm{V}}\right.$, $T)$ to $S$. Then $U_{i}$ can eavesdrop on the login request of the valid user $\mathrm{U}_{\mathrm{V}}$. $\mathrm{U}_{\mathrm{i}}$ computes $\mathrm{h}\left(\mathrm{x} \| \mathrm{y}_{\mathrm{V}}\right)=\mathrm{CID}_{\mathrm{V}} \oplus \mathrm{h}(\mathrm{h}(\mathrm{x}) \| \mathrm{T})$, $\mathrm{CID}^{\prime}=\mathrm{h}\left(\mathrm{x} \| \mathrm{y}_{\mathrm{V}}\right) \oplus \mathrm{h}\left(\mathrm{h}(\mathrm{x}) \| \mathrm{T}^{\prime}\right)$ where $\mathrm{T}^{\prime}$ is the current date and time of $U_{i} \cdot U_{i}$ computes $M^{\prime}=\left(h\left(h(x)\left\|h\left(x \| y_{V}\right)\right\| T^{\prime}\right)\right.$. Here CID' and $M^{\prime}$ are $U_{i}$ 's version of $C^{\prime} D_{V}$ and $M_{V}$ respectively. $U_{i}$ sends (CID', M', T') to the server. In this case, $\mathrm{T}$ ' is valid since it is computed by $U_{i}$ with the current date and time. Upon receiving the login request, $S$ verifies the validity of $T$ '. If true, $\mathrm{S}$ accepts the request and computes $\mathrm{A}_{\mathrm{i}}{ }^{*}=\mathrm{h}\left(\mathrm{x} \| \mathrm{y}_{\mathrm{i}}\right)^{*}=$ $h\left(x \| y_{V}\right)=C I D^{\prime} \oplus h\left(h(x) \| T^{\prime}\right)$. S retrieves $y_{V} \oplus x$ and $I_{V}$ $\oplus \mathrm{h}(\mathrm{x})$ corresponding to $\mathrm{A}_{\mathrm{i}}=\mathrm{A}_{\mathrm{i}}^{*}$ from its database. $\mathrm{S}$ extracts $\mathrm{y}_{\mathrm{V}}$ from $\mathrm{y}_{\mathrm{V}} \oplus \mathrm{x}$ and $\mathrm{ID}_{\mathrm{V}}$ from $\mathrm{ID}_{\mathrm{V}} \oplus \mathrm{h}(\mathrm{x})$, since it knows $\mathrm{x}$. $\mathrm{S}$ computes $\mathrm{M}_{\mathrm{V}}{ }^{*}=\mathrm{h}\left(\mathrm{h}(\mathrm{x})\left\|\mathrm{A}_{\mathrm{i}}\right\| \mathrm{T}^{\prime}\right)$ and compare $\mathrm{M}_{\mathrm{V}}{ }^{*}$ with $\mathrm{M}^{\prime}$ in the received login request. Here the condition will surely hold and $S$ will accept the login request. $S$ computes $V_{i}=h\left(A_{i} \|\right.$ $\left.h(x)\left\|T_{s}\right\| T^{\prime}\right)$, where $T_{s}$ is the server's current date and time. $\mathrm{S}$ sends $\left(\mathrm{V}_{\mathrm{i}}, \mathrm{T}_{\mathrm{s}}\right)$ to the smart card of valid user and $\mathrm{U}_{\mathrm{i}}$ intercepts the same. The malicious valid user $U_{i}$ impersonates his peer valid user $U_{L}$ and he can carry forward the communication if he can find the ID of $\mathrm{U}_{\mathrm{L}}$, and generate the session key.

\subsection{Offline Password Guessing Attack}

In case a malicious valid user $U_{i}$, gets the smart card of another registered user $U_{k}$, then he will be able to extract its contents. Then if $U_{i}$ intercepts a previous login request, $\left(\mathrm{CID}_{\mathrm{k}}\right.$, $\left.\mathrm{M}_{\mathrm{k}}, \mathrm{T}\right)$ of $\mathrm{U}_{\mathrm{k}}$, he can extract $\mathrm{h}\left(\mathrm{x} \| \mathrm{y}_{\mathrm{k}}\right)$ from $\mathrm{CID}_{\mathrm{k}}$ as $\mathrm{h}\left(\mathrm{x} \| \mathrm{y}_{\mathrm{k}}\right)=$ $\mathrm{CID}_{\mathrm{k}} \oplus \mathrm{h}(\mathrm{h}(\mathrm{x}) \| \mathrm{T})$ since he knows $\mathrm{h}(\mathrm{x})$ and $\mathrm{T}$. Now $\mathrm{U}_{\mathrm{i}}$ can calculate $\mathrm{h}\left(\mathrm{P}_{\mathrm{k}}\right)=\mathrm{C}_{\mathrm{k}} \oplus \mathrm{h}\left(\mathrm{x} \| \mathrm{y}_{\mathrm{k}}\right)$ since he has extracted $\mathrm{C}_{\mathrm{k}}$ from the smart card and $h\left(x \| y_{k}\right)$ from the login request message respectively of user $U_{k}$. He can guess the value of $P_{k}$ to be $P_{k}$ * from a dictionary space $\mathrm{D}_{\mathrm{pw}}$. Computes $\mathrm{h}\left(\mathrm{P}_{\mathrm{k}} *\right)$ and compares it with $\mathrm{h}\left(\mathrm{P}_{\mathrm{k}}\right)$. The process is repeated until a match is found.

\section{PROPOSED SCHEME}

The vulnerabilities of Sood et al.'s scheme is mainly due to the fact that the valid user can easily calculate the values of $h\left(x \| y_{i}\right)$ and $h(x)$ as computed by the server $S$ and this should be prevented. Also the verification table makes the scheme vulnerable to stealing information from database attack and ID-theft attack. Taking into consideration the above mentioned vulnerabilities a remote user authentication scheme that eliminates the requirement of verification table is proposed. The scheme which attempts to address the flaws of Sood et al.'s scheme consists of four phases' viz., Initialization, Registration, Login \& and Password change phase as summarized in figure 1. The notations used are listed in Table1.

\section{Table 1. Notations}

\begin{tabular}{|l|l|}
\hline $\mathrm{U}_{\mathrm{i}}, \mathrm{S}$ & $\mathrm{i}^{\text {th }}$ User, Server \\
\hline $\mathrm{ID}_{\mathrm{i}}, \mathrm{P}_{\mathrm{i}}$ & $\begin{array}{l}\text { Unique Identification of } \mathrm{U}_{\mathrm{i}}, \text { Password of } \\
\text { user } \mathrm{U}_{\mathrm{i}}\end{array}$ \\
\hline $\mathrm{Z}_{\mathrm{n}}$ & Additive Cyclic group, integer modulo ' $\mathrm{l}$ \\
\hline $\mathrm{g} 0$ & Generator of cyclic group \\
\hline $\mathrm{a}, \mathrm{b}_{\mathrm{i}}$ & $\begin{array}{l}\text { Server's secret key, Random number } \\
\text { selected by } \mathrm{S} \text { unique to each } \mathrm{U}_{\mathrm{i}}\end{array}$ \\
\hline $\mathrm{r}$ & $\begin{array}{l}\text { Random number generated by smart card } \\
\text { unique to each session }\end{array}$ \\
\hline$\oplus, \|$ & $\begin{array}{l}\text { One way hash function, XOR operation, } \\
\text { Concatenation Operation }\end{array}$ \\
\hline$\Rightarrow$ & Secure Communication Channel \\
\hline
\end{tabular}

\subsection{Initialization Phase}

Given a finite additive cyclic group $Z_{\mathrm{n}}=\left\langle\mathrm{g}_{0}\right\rangle$, of order $\mathrm{q}$ where $\mathrm{q}$ is a prime number. Element $\mathrm{g}_{0}$ is a generator of $\mathrm{Z}_{\mathrm{n}}$ and is kept as a secret between the user and the server.

\subsection{Registration Phase}

A user $U_{i}$ who wants to become a registered member of the system chooses her identity $\mathrm{ID}_{\mathrm{i}}$ and password $\mathrm{P}_{\mathrm{i}}$. $\mathrm{U}_{\mathrm{i}}$ computes $\mathrm{x}=\mathrm{h}\left(\mathrm{P}_{\mathrm{i}}\right), \mathrm{y}=\mathrm{g}_{0}^{\mathrm{x}}, \mathrm{h}\left(\mathrm{ID}_{\mathrm{i}}\right)$ and submits $\left(\mathrm{h}\left(\mathrm{ID}_{\mathrm{i}}\right), \mathrm{y}\right)$ to server through a secure communication channel. Server $S$ chooses the value ' $a$ ' as its secret key and a random number ' $b_{i}$ ' where $\mathrm{a}, \mathrm{b}_{\mathrm{i}} \in \mathrm{Z}_{\mathrm{n}}$ and computes the following: $\mathrm{A}_{\mathrm{i}}=\mathrm{h}\left(\mathrm{a}|| \mathrm{b}_{\mathrm{i}}\right) ; \mathrm{B}_{\mathrm{i}}=$ $\mathrm{h}\left(\mathrm{h}\left(\mathrm{ID}_{\mathrm{i}}\right) \| \mathrm{y}\right) \oplus \mathrm{y} \oplus \mathrm{h}\left(\mathrm{a} \| \mathrm{b}_{\mathrm{i}}\right) ; \mathrm{C}_{\mathrm{i}}=\mathrm{h}\left(\mathrm{a} \| \mathrm{b}_{\mathrm{i}}\right) \oplus \mathrm{y} ; \mathrm{D}_{\mathrm{i}}=\mathrm{h}\left(\mathrm{h}\left(\mathrm{ID}_{\mathrm{i}}\right)\right.$ $\| y) \oplus h(a) . S \Rightarrow S C:\left(B_{i}, C_{i}, D_{i}\right.$ and $\left.h().\right)$. $U_{i}$ stores $g_{0}$ in the smart card which now contains, $\left(B_{i}, C_{i}, D_{i}, g_{0}\right.$ and $\left.h().\right)$ 


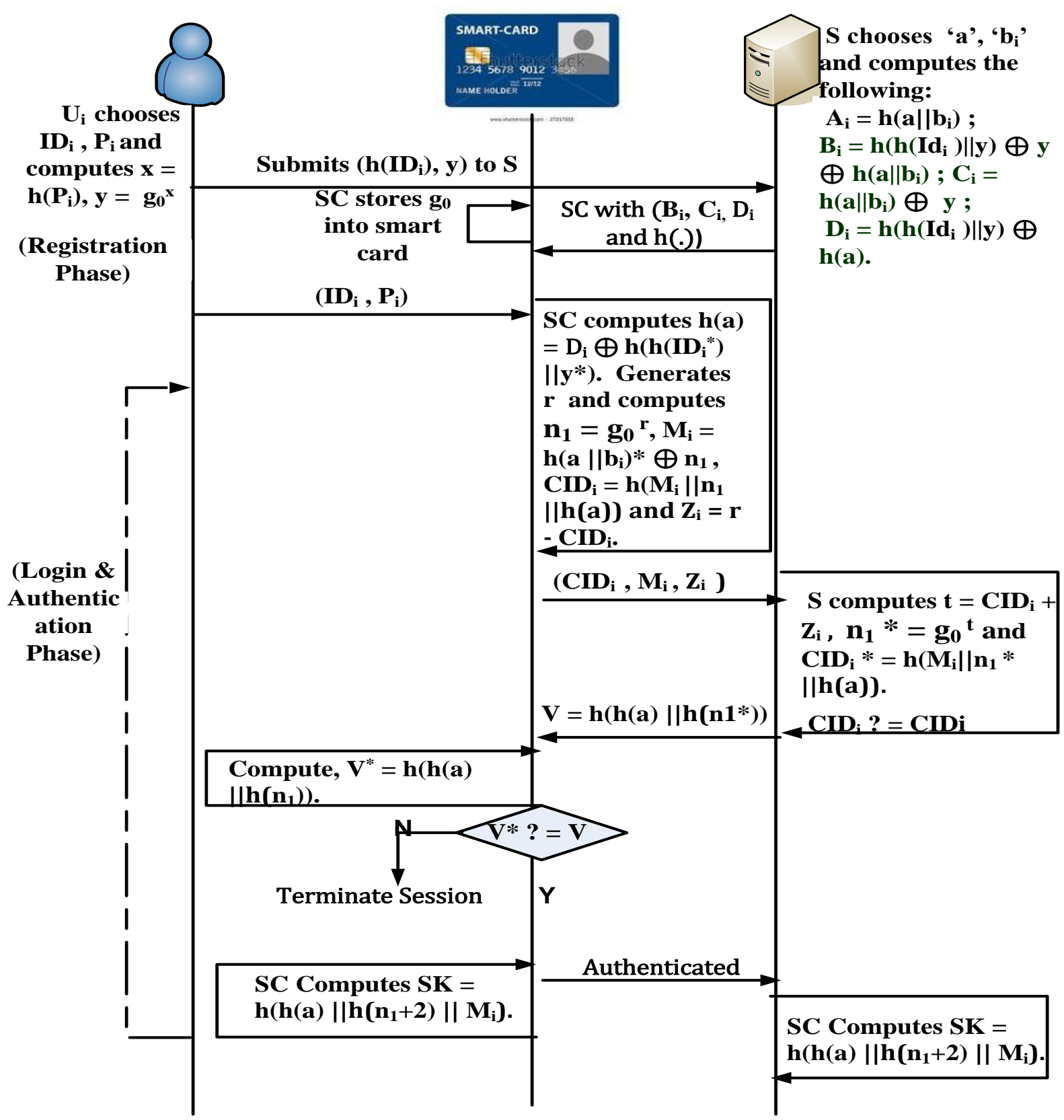

Fig 1: Phases of Proposed Protocol

\subsection{Login and Authentication Phase}

Whenever a registered user $U_{i}$ wants to login, he inserts his SC into the reader and inputs $\mathrm{ID}_{\mathrm{i}}{ }^{*}$ and $\mathrm{P}_{\mathrm{i}}{ }^{*}$. Then SC computes $\mathrm{x}^{*}=\mathrm{h}\left(\mathrm{P}_{\mathrm{i}}^{*}\right)$ and $\mathrm{y}^{*}=\mathrm{g}_{0}{ }^{* *}, \mathrm{~A}_{\mathrm{i}}{ }^{*}=\mathrm{h}\left(\mathrm{a} \| \mathrm{b}_{\mathrm{i}}\right)^{*}=\mathrm{C}_{\mathrm{i}} \oplus \mathrm{y}^{*}, \mathrm{~B}_{\mathrm{i}}{ }^{*}=$ $\mathrm{h}\left(\mathrm{h}\left(\mathrm{ID}_{\mathrm{i}}^{*}\right) \| \mathrm{y}^{*}\right) \oplus \mathrm{y}^{*} \oplus \mathrm{h}\left(\mathrm{a} \| \mathrm{b}_{\mathrm{i}}\right)^{*} . \mathrm{SC}$ checks $\mathrm{B}_{\mathrm{i}}^{*}=\mathrm{B}_{\mathrm{i}}$. If the equality does not hold, $\mathrm{SC}$ rejects the request. Otherwise, the identity of the $U_{i}$ is validated and the SC computes the login request as follows and sends it to the server. SC computes h(a) $=D_{i} \oplus h\left(h\left(I_{i}^{*}\right) \| y^{*}\right)$. SC generates a random number $r \in Z_{n}$ and computes $n_{1}=g_{0}{ }^{r}, M_{i}=h\left(h\left(a \| b_{i}\right)^{*} \| r \oplus n_{1}\right), C I_{i}=$ $\mathrm{h}\left(\mathrm{M}_{\mathrm{i}}\left\|\mathrm{n}_{1}\right\| \mathrm{h}(\mathrm{a})\right)$ and $\mathrm{Z}_{\mathrm{i}}=\mathrm{r}-\mathrm{CID}_{\mathrm{i}}$. Then $\mathrm{SC} \Rightarrow \mathrm{S}:\left(\mathrm{CID}_{\mathrm{i}}, \mathrm{M}_{\mathrm{i}}\right.$, $\mathrm{Z}_{\mathrm{i}}$ ). In this phase, there is a limit on the number of attempts a user can make to login to the server after which, the smart card gets locked.

\subsection{Password Change Phase}

A registered user can change the password at his will, without the intervention of the server. $\mathrm{U}_{\mathrm{i}}$ inputs $\mathrm{ID}_{\mathrm{i}}{ }^{*}$ and $\mathrm{P}_{\mathrm{i}}{ }^{*}$. SC computes $\mathrm{x}^{*}=\mathrm{h}\left(\mathrm{P}_{\mathrm{i}}^{*}\right), \mathrm{y}^{*}=\mathrm{g}_{0}{ }^{\mathrm{x}^{*}}, \mathrm{~h}\left(\mathrm{a} \| \mathrm{b}_{\mathrm{i}}\right)^{*}=\mathrm{C}_{\mathrm{i}} \oplus \mathrm{y}^{*}, \mathrm{~B}_{\mathrm{i}}^{*}=$ $\mathrm{h}\left(\mathrm{h}\left(\mathrm{ID}_{\mathrm{i}}^{*}\right) \| \mathrm{y}^{*}\right) \oplus \mathrm{y}^{*} \oplus \mathrm{h}\left(\mathrm{a} \| \mathrm{b}_{\mathrm{i}}\right)^{*} . \mathrm{SC}$ compares $\mathrm{B}_{\mathrm{i}}^{*}=\mathrm{B}_{\mathrm{i}}$. If the equality does not hold, $\mathrm{SC}$ rejects the request. If the verification holds, the identity $\mathrm{U}_{\mathrm{i}}$ is validated and $\mathrm{SC}$ prompts the user to enter the new password $\mathrm{P}_{\mathrm{i}}^{\text {new }}$. Then $\mathrm{SC}$ computes $\mathrm{x}_{\text {new }}=\mathrm{h}\left(\mathrm{P}_{\mathrm{i}}^{\text {new }}\right), \mathrm{y}_{\text {new }}=\mathrm{g}_{0}{ }^{\text {Xnew }}, \mathrm{h}(\mathrm{a})^{*}=\mathrm{D}_{\mathrm{i}} \oplus \mathrm{h}\left(\mathrm{h}\left(\mathrm{ID}_{\mathrm{i}}^{*}\right) \| \mathrm{y}\right) . \mathrm{SC}$ computes $B_{i}{ }^{\text {new }}=h\left(h\left(I_{i}{ }^{*}\right) \| y_{\text {new }}\right) \oplus y_{\text {new }} \oplus h\left(a \mid l b_{i}\right)^{*}, C_{i}{ }^{\text {new }}$ $=h\left(a \| b_{i}\right)^{*} \oplus y_{\text {new }}, D_{i}^{\text {new }}=h\left(h\left(I_{i}^{*}\right) \| y_{\text {new }}\right) \oplus h(a) *$ SC updates the values of $B_{i}, C_{i}$ and $D_{i}$ stored in the memory of the smart card with the values $B_{i}{ }^{\text {new }}, C_{i}^{\text {new }}, D_{i}{ }^{\text {new }}$.

\section{SECURITY ANALYSIS}

This section examines the resistance of the proposed scheme to various possible attacks on remote user authentication scheme.

\subsection{Impersonation Attack}

This attack attempts to forge the message by using the information obtained from the target authentication scheme. 
When forging the message, the attacker disguises as a legal user. The attacker attempts to modify the message $\left(\mathrm{CID}_{\mathrm{i}}, \mathrm{M}_{\mathrm{i}}\right.$, $\mathrm{Z}_{\mathrm{i}}$ ). But the attacker needs to know the secret key ' $\mathrm{a}$ ' of the server, random number ' $b_{i}$ ' chosen by server uniquely for user $\mathrm{U}_{\mathrm{i}}$ and the client's random number ' $\mathrm{r}$ ' and nonce ' $\mathrm{n}_{1}$ ', to compute the valid values of $\mathrm{CID}_{\mathrm{i}}, \mathrm{M}_{\mathrm{i}}, \mathrm{Z}_{\mathrm{i}}$. Otherwise the attempts would be rejected at the authentication phase. Additionally the disguise attack is impossible because' r'is a session variable which is valid only for a single session and the session ID's are well protected to prevent a session hijacking attack. Moreover, the attacker should know ' $n_{1}$ ' to calculate the session key to carry forward the communication. Hence the proposed scheme is secure against, forgery or impersonation attack.

\subsection{Insider Attack}

In the registration phase, the user submits $\mathrm{y}=\mathrm{g}_{0} \mathrm{x}$, where $\mathrm{x}=$ $\mathrm{h}$ (password) to the server. So, even an insider cannot know the password of a user, since computing $\mathrm{x}$ from $\mathrm{y}$ involves the solving the discrete logarithm problem. Here $\mathrm{g}_{0}$ is a generator of the additive cyclic group $Z_{n}$, where $Z_{n}$ is group of integer's modular ' $n$ '. Calculating the discrete logarithm of values generated using modular arithmetic is a computationally intensive task which makes the scheme secure against insider attack.

\subsection{Stolen Verifier Attack}

The proposed scheme does not require the server to maintain a verification table. Hence this attack which involves copying the authentication parameters stored in a verifier table and using the same to impersonate a valid user does not have any relevance here.

\subsection{Time Concurrency Weaknesses}

Sood et al.'s scheme uses time stamps to avoid replay attacks. Time stamps require proper synchronization of clocks at the ends of both the serer and the client and this may have practical difficulties when the client and server belong to different time zones.. The proposed scheme overcomes this limitation by using nonce values.

\subsection{Security against Denial of Service \\ Attack}

The proposed scheme prevents unauthorized modification of password verification information by enabling the smart card to check the validity of user before updating the password. It is impossible to correctly guess $\operatorname{ID}_{\mathrm{i}}$ and $\mathrm{P}_{\mathrm{i}}$ both simultaneously even after getting the SC of the legitimate user. Also an administrator cannot modify the password information in database, since there is no verification table maintained by the server.

\subsection{Offline Password Guessing Attack}

In this attack an attacker first tries to obtain some client or server verification information containing the password parameter and tries to guess the password offline. In the proposed scheme the password ' $\mathrm{P}_{\mathrm{i}}$ ' is neither transmitted nor stored in the smart card in its bare form. The password is stored and transmitted as $\mathrm{y}=\mathrm{g}_{0}{ }^{\mathrm{x}}$, where $\mathrm{x}=\mathrm{h}\left(\mathrm{P}_{\mathrm{i}}\right)$. To get the password ' $\mathrm{x}$ ' the attacker should calculate the discrete logarithm of ' $y$ ' to the base $g_{0}$ and calculation of discrete logarithms in modular arithmetic is a computationally intensive and time consuming process. Thus the complexity involved in solving discrete logarithmic problem, makes the proposed scheme secure against offline password guessing attack.

\subsection{Attack on User Anonymity}

In Sood et al.'s scheme, the attacker is able to easily extract $\mathrm{h}\left(\mathrm{x} \| \mathrm{y}_{\mathrm{i}}\right)$, from the login request message. This value which is unique to a particular user, will be the same in all login request messages providing the attacker to trace the messages as belonging to a particular user. In the proposed scheme, even though the unique value $h\left(a|| b_{i}\right)$ is used in the login request message, it cannot be extracted by the attacker. This prevents him from tracing out the valid user and preserves user's anonymity.

\section{COST ANALYSIS}

The comparison of the proposed scheme with Sood et al.'s scheme is summarized in Table 2 and 3 respectively. This scheme assumes that the parameters $\operatorname{ID}_{\mathrm{i}}, \mathrm{P}_{\mathrm{i}}, \mathrm{a}, \mathrm{b}_{\mathrm{i}}, \mathrm{r}$ are 128-bit long. We use SHA-2 a more secure hash function whose output is 256 bits long.

Table 2. Functionality Comparison

\begin{tabular}{|c|c|c|c|c|}
\hline $\begin{array}{c}\text { Proposed } \\
\text { Scheme }\end{array}$ & $\begin{array}{l}\text { Sood } \\
\text { et } \\
\text { al.[19] }\end{array}$ & $\begin{array}{c}\text { Liao- } \\
\text { Wang[11] }\end{array}$ & $\begin{array}{c}\text { Hsiang- } \\
\text { Shih[18] }\end{array}$ \\
\hline $\begin{array}{c}\text { Resists } \\
\text { Attack }\end{array}$ & Yes & No & No & No \\
\hline $\begin{array}{c}\text { Resists } \\
\text { Password } \\
\text { Guessing } \\
\text { Attack }\end{array}$ & Yes & No & Yes & No \\
\hline $\begin{array}{c}\text { Overcomes } \\
\text { Time } \\
\begin{array}{c}\text { Concurrency } \\
\text { Weaknesses }\end{array}\end{array}$ & Yes & No & Yes & Yes \\
\hline $\begin{array}{c}\text { Resists } \\
\text { Denial-of- } \\
\text { Service } \\
\text { Attack }\end{array}$ & Yes & No & Yes & Yes \\
\hline $\begin{array}{c}\text { Resists Stolen } \\
\text { Verifier } \\
\text { Attack }\end{array}$ & Yes & No & Yes & No \\
\hline $\begin{array}{c}\text { Insider Attack } \\
\text { Agreement }\end{array}$ & Yes & No & No & Yes \\
\hline $\begin{array}{c}\text { Provides } \\
\text { Mutual } \\
\text { Authentication }\end{array}$ & Yes & Yes & yes & yes \\
\hline Provides & Yes & Yes & yes & Yes \\
\hline
\end{tabular}

Let $\mathrm{T}_{\mathrm{H}}$ and $\mathrm{T}_{\mathrm{E}}$ denote the time complexity for hashing, exponentiation and symmetric key encryption respectively. In the proposed scheme, the parameters stored in the smart card are $\mathrm{B}_{\mathrm{i}}, \mathrm{C}_{\mathrm{i}}, \mathrm{D}_{\mathrm{i}}$ and $\mathrm{g}_{0}$ and hence $\mathrm{E} 1$, the memory needed in the smart card is 896 bits. Communication cost of authentication (E2) includes the capacity of transmitting message involved in the authentication. The capacity of transmitting message $\left(\mathrm{CID}_{\mathrm{i}}\right.$ , $\mathrm{M}_{\mathrm{i}}, \mathrm{Z}_{\mathrm{i}}$ ) and $\mathrm{V}_{\mathrm{i}}$ is 1024 bits. The computation cost of registration (E3) is the total time of all operations executed in this phase. The computation cost of the user (E4) and the service provider (E5) is the time spent by the user and 
the service provider during the process of login, authentication and session key agreement.

Table 3. Cost Comparison of Proposed Scheme and Sood et al.'s Scheme

\begin{tabular}{|l|l|l|}
\hline & Proposed Scheme & Sood et al.[19] \\
\hline E1 & $896(=3 * 256+128)$ bits & 384 bits \\
\hline E2 & $1024(=4 * 256)$ bits & 640 bits \\
\hline E3 & $\mathrm{T}_{\mathrm{E}}+6 \mathrm{~T}_{\mathrm{H}}$ & $4 \mathrm{~T}_{\mathrm{H}}$ \\
\hline E4 & $2 \mathrm{~T}_{\mathrm{E}}+5 \mathrm{~T}_{\mathrm{H}}$ & $6 \mathrm{~T}_{\mathrm{H}}$ \\
\hline E5 & $\mathrm{T}_{\mathrm{E}}+3 \mathrm{~T}_{\mathrm{H}}$ & $5 \mathrm{~T}_{\mathrm{H}}$ \\
\hline
\end{tabular}

\section{CONCLUSION}

In 2006, Liou et al. proposed a password authentication scheme using smart cards ,to overcome the security limitations of the dynamic ID based authentication scheme proposed by Das et al. However, in 2010, Sood et. al., demonstrated that Liou at al.' scheme is not resistant to malicious user attack, man in the middle attack, impersonation attack and offline password guessing attack and they proposed a modification. This paper analyzes the weaknesses of Sood at al.'s scheme and proposes a modified version without verification table. The proposed scheme enhances security by using cyclic groups as the domain for generating the security parameters and by exploiting the difficulty in solving discrete logarithmic problem in modular arithmetic. Future scope of this work is to increase efficiency by reducing the computation cost and enhance security by making the protocol resistant to more attacks.

\section{REFERENCES}

[1] W.C. Ku, S.M. Chen, "Weakness and Improvement of an Efficient Password Based Remote User Authentication Scheme Using Smart Cards," IEEE Transactions on Consumer Electronics 50(1), 204-207 (2004)

[2] Y.C. Chen, L.Y. Yeh, " An Efficient Nonce-Based Authentication Scheme with Key Agreement," Applied Mathematics and Computation 169(2), 982-994 (2005)

[3] W.G. Shieh, J.M. Wang, "Efficient Remote Mutual Authentication and Key Agreement," Computers and Security 25(1), 72-77 (2006)

[4] H.C Hsiang, W.K. Shih,"Weakness and Improvement of Yoon-Ryu-Yoo Remote User Authentication Scheme Using Smart Cards," Computer Communications 32(4), 649-652 (2009)

[5] M.Kumar, "A New Secure Remote User Authentication Scheme with Smart Cards", International Journal of Network Security 11, 88-93 (2010)

[6] S.K. Sood, A.K Sarje, K.Singh, "A Secure Dynamic Identity-Based Remote User Authentication Scheme," In:T. Janowski, H. Mohanty. (eds) ICDCIT 2010. LNCS, vol. 5966, pp.224-235, Springer, Heidelberg (2010)]

[7] M.Misbahuddin, A.A. Mohammed, M.H. Shastri, "A Simple and Efficient Solution to Remote User Authentication using Smart Cards," In Proceeding of International Conference on Innovations in Information Technology (IIT ’06), Dubai,Nov.2006, pp.1-5.
[8] L. Lamport, "Password Authentication with Insecure Communication," Communications of the ACM, vol. 24. no. 11, pp. 770-772, November 1981

[9] M.L. Das, A. Saxena and V.P. Gulati," A Dynamic IDBased Remote User Authentication Scheme, "IEEE Transactions on Consumer Electronics, vol. 50, no.2, pp. 629-631, May 2004

[10] T. Goriparthi, M.L. Das, A.Saxena, "An Improved Bilinear Pairing Based Remote User Authentication Scheme," Computer Standards and Interfaces 2009, 31 : (181-185)

[11] Y.P. Liao, S.S. Wang, “A Secure Dynamic ID Based Remote User Authentication Scheme for Multi Server Environment," Computer Standards and Interfaces 2009, 31(1): $24-29$

[12] Y.Y. Wang, J.Y. Liu, F.X. Xiao, J.Dan, "A More Efficient and Secure Dynamic ID Based Remote User Authentication Scheme," Computer Communications, 2009, 32(2): 583-585

[13] H.Y Chien and C.H. Chen, "A Remote Authentication Scheme Preserving User Anonymity," In Proceedings of Advanced Information Networking and Applications, vol.2, pp. 245-248, March 2005

[14] I.E. Liao, C.C.Lee and M.S. Hwang, "Security Enhancement for a Dynamic ID-Based Remote User Authentication Scheme," In Proceeding of Conference on Next Generation on Web Services Practice, pp. $437-$ 440, July 2005

[15] [15]

E.J. Yoon and K.Y. Yoo, "Improving the Dynamic ID-Based Remote Mutual Authentication Scheme," In Proceedings of OTM Workshops 2006, LNCS 4277, pp. 499-507, July 2006

[16] Y.P Liou, J.Lin and S.S. Wang, ” A New Dynamic IDBased Remote User Authentication Scheme Using Smart Cards," In Proceedings of $16^{\text {th }}$ Information Security Conference, Taiwan, pp. 198-205, July 2006

[17] H.C. Shih, "Cryptanalysis on Two Password Authentication Schemes," Laboratory of Cryptography and Information Security, National Central University, Taiwan, July 2008

[18] H.C. Hsiang and W.K. Shih, "Improvement of the Secure Dynamic ID Based Remote User Authentication Scheme for Multi-Server Environment", Computer Standards \& Interfaces, vol.31, (2009), pp.1118-1123

[19] S.K. Sood. A.K. Sarje and K.Singh, "An Improvement of Liou et al.'s Authentication Scheme Using Smart Cards," International Journal of Computer Applications, vol.1, no.8, (2010), pp.16-23

[20] W.C. Ku and S.T. Chang, "Impersonation Attack on Dynamic ID-Based Remote User Authentication Scheme using Smart Cards," IEICE Transactions on Communications, vol. E88-B, No.5, pp.2165-2167, May 2005

[21] C.S. Kwang and G.C. Jung, "An Improvement of Sood et al.'s Authentication Scheme using Smart Card", International Journal of Security and Its Applications, vol. 7, No. 3, May, 2013 